\title{
El comunicador corporativo como sujeto político en las Pymes de Medellín
}

\author{
al \\ Carlos Alberto Galvis Ortiz * \\ Luis Horacio Botero Montoya
}

Recibido: 11 de noviembre de 2009

Aceptado: 25 de noviembre de 2009

\section{RESUMEN}

Analizar el impacto y el accionar de los egresados del programa de Comunicación y Relaciones Corporativas como sujetos políticos en las pequeñas y medianas empresas -pymes-, ubicadas en el Municipio de Medellín, en el período comprendido entre el 2002-2006, constituye un reto para los propósitos investigativos de las facultades de comunicación y, en particular, para los propósitos del grupo de investigación en comunicación y relaciones corporativas -Corpus-, adscrito a la Facultad de Comunicación de la Universidad de Medellín.

Este artículo pretende explorar, desde la fundamentación teórica que sustenta el Programa de Comunicación y Relaciones Corporativas, los resultados del impacto generado por los egresados y su papel como sujetos políticos en las pequeñas y medianas empresas analizadas.

Además, y dado el auge de los programas de Comunicación Social-Periodismo y de programas de Comunicación, en general, las empresas pequeñas y medianas -pymes- están demandando cada vez más egresados de comunicación a fin de que este tipo de profesional intervenga, en forma directa, sus procesos empresariales no sólo desde el ámbito de la información, sino desde los conocimientos y habilidades comunicativas como apoyo a la labor gerencial.

En este artículo, que recoge algunos de los elementos más relevantes de la investigación terminada "Impacto de los egresados del programa de Comunicación y Relaciones Corporativas en las pequeñas y medianas empresas, ubicadas en el municipio de Medellín, período 2002-2006" del grupo CORPUS, pretendemos establecer el alcance real del impacto laboral, social y político de los egresados del programa de Comunicación y Relaciones Corporativas, así como reflexionar sobre la necesaria relación entre la educación y la comunicación y su potencialidad para generar aprehensiones democráticas en los actores-comunicadores que interactúan en entornos complejos.

Palabras clave: Democracia, ciudadanía, educación, comunicación

* Carlos Alberto Galvis Ortiz. Colombia. Comunicador Social y Periodista de la Universidad Jorge Tadeo Lozano de Bogotá. Licenciado en Español y Literatura de la Universidad del Quindío. Magíster en Educación (énfasis en Democracia) de la Universidad Católica de Manizales. Profesor de tiempo completo de la Facultad de Comunicación de la Universidad de Medellín. Coordinador del Grupo de Investigación en Comunicación Corpus, categoría C de Colciencias. E-mail: cagalvis@gmail.com

** Luis Horacio Botero Montoya. Colombia. Comunicador Social-Periodista de la Universidad de Antioquia. Especialista en Gestión Pública de la Escuela Superior de Administración Pública; especialista en Dirección Empresarial y en Gerencia de la Fundación Universitaria Ceipa, Executive MBA de la Escuela de Administración de Empresas -EAE- de Barcelona-España y magíster en Ciencias de la Administración de la Universidad Eafit-HEC de Canadá. Docente-investigador del grupo de Investigación en Comunicación Corpus de la Universidad de Medellín y clasificado en categoría C de Colciencias. E-mail: 1hbotero@udem.edu.co 


\title{
A corporate communicator as a political subject in Medellín small and medium size companies
}

\begin{abstract}
To analyze the impact and actions of graduates of Communication and Corporate Relations program of studies as political subjects in small and medium size enterprises -SMEs-, located in Medellín municipality, from 2002 to 2006, is a challenge for communication department research purposes, specifically, for communication and corporate relations research group -Corpus, which belong to Universidad de Medellín Communication School.

This article aims to explore, from the theoretical foundation that supports the Communication and Corporate Relations Program of studies, results of the impact caused by the graduates of this program and their role as political actors in the small and medium enterprises studied.

Furthermore, and given the rise of social communication, journalism, and communication programs of study, in general, small and medium size enterprises -SMEs- are demanding more and more graduates in communication, so that this type of professional may, directly, participate in their business processes, not only from the field of information, but from the knowledge and communication skills as support to the managerial work.

This article, which collects some of the most important elements of the above mentioned research, intends to establish the real impact of Communication and Corporate Relations program of studies, and also aims to reflect on the necessary relationship between education and communication and their potential to generate democratic apprehensions in the actors -communicators- who interact in complex environments.
\end{abstract}

Key words: Democracy, citizens, education, communication 
La educación debe contribuir a la autoformación de la persona (aprender y asumir la condición humana, aprender a vivir) y aprender a convertirse en un ciudadano. Un ciudadano, en una democracia, se define por su solidaridad y su responsabilidad. (...) La enseñanza puede intentar eficazmente hacer converger las ciencias naturales, las ciencias humanas, la cultura de las humanidades y la filosofía hacia el estudio de la condición humana.

EDGar Morin

\section{A MODO DE ESCOLIO}

La educación y la comunicación están lejos de ser instrumentos de conocimiento perfecto, para aprehender la realidad. Sin embargo, es lo mejor que tenemos.

La educación y la comunicación por sí mismas no resuelven nada. Se requiere del sujeto-actor, de su actitud política y democrática que lo comprometa a una transformación personal permanente y continua y que, a su vez, redunde en construcción de sociedad ${ }^{1}$.

De allí que devenir ciudadano o, mejor, demócrata, es un trabajo arduo y dinámico que requiere de mentes formadas en la complejidad, el escepticismo y el asombro. Complejidad para entender los múltiples cruces rizomáticos de una sociedad que, de forma permanente, se transforma dentro del caos y la incertidumbre; escepticismo como herramienta para desvelar el camino a la verdad, y asombro para maravillarnos por el descubrimiento.

\footnotetext{
La democracia no sólo es un imperativo, sino una necesidad para la tríada republicana de libertad, igualdad y fraternidad, pregonada en la Revolución Francesa. Se sugiere que el lector vaya al texto Comunicación pública. Repensar la comunicación para la democracia, capítulo XI, de los autores Luis Horacio Botero Montoya y Carlos Galvis y publicado por el Sello Editorial de la Universidad de Medellín en junio de 2009, ISBN 978-958-8348-51-3.
}

Frente a ese rol democrático, como su nombre lo indica, se profesan actos de fe en el ser humano y la sociedad. Decía el filósofo francés J. Derridá ${ }^{2}$, que "profesar es dar una prueba comprometiendo nuestra responsabilidad". Los filósofos griegos enunciaban la determinación de philosophiam profiteri, de profesar la filosofía.

No simplemente ser un ciudadano del común, sino comprometerse, mediante una promesa pública, a consagrarse a la democracia, a dar testimonio de ella, e incluso, a emanciparse por ella.

En este sentido, la educación y la comunicación son componentes fundamentales, para forjar ciudadanos (léase profesionales universitarios) en la triple racionalidad de lo ético, lo estético y lo científico.

Sin embargo, la humanidad enfrenta un gran desafío en la modernidad: la perversidad del desarrollo, concebido desde una racionalidad técnico-científica, basada en el progreso, como crecimiento económico, y la industrialización, como garantes de un mejor vivir, donde se actúa de acuerdo con una lógica de producción de bienes, más que de bienestar para la sociedad, bajo los parámetros de un eficientismo que reduce al sujeto a un actor al servicio de un llamado desde la visión economicista de productividad y competitividad.

El capitalismo, sistema donde se actualiza esta visión instrumental del mundo, dejó de ser una posibilidad para la búsqueda de una nueva relación más humana, toda vez que éste se ha reducido a un capitalismo ficticio, marcado más por una especulación financiera que por un capitalismo industrial real, capaz de transformar y transformarse para mejorar la relación de los sujetos-actores y no de los consumidores-pasivos en lo que nos convirtió el capitalismo actual.

2 Para mayor información sobre este autor y su propuesta, se sugiere leer "La Universidad sin condición", publicado por Editorial Trotta, Madrid, 2002. 
El premio Nobel de economía del 2001 y ex funcionario del Banco Mundial, Joseph Stiglitz, en su libro titulado Cuando el capitalismo pierde la cabeza, señala que el capitalismo financiero a la americana, en tanto que sistema político-económico, está en grave crisis. Este sistema, se ha convertido, "en un sistema de capitalismo de amigotes y de integristas fanáticos del mercado" (Stiglitz, 2003).

Hoy, a raíz de esta lógica mecanicista e instrumental que privilegia la forma y no el fondo y reproduce una negación de la humanización necesaria, y a pesar de los avances científicos y tecnológicos obtenidos, vemos los efectos devastadores para la humanidad en todas sus esferas, que nos llevan a una incertidumbre acerca del futuro y a una incapacidad de comprehender e incidir en los problemas, debido a una fragmentación y parcelación del conocimiento que nos ha conducido a conocer una parte específica y abstracta de la realidad y, lo que es peor, totalmente descontextualizada de aquélla.

Por eso, y tal como lo afirma el filósofo Edgar Morin, "la educación hoy debe esforzarse por practicar un pensamiento que se desvele sin cesar por contextualizar y totalizar las informaciones y los conocimientos, que se apliquen a luchar contra el error y la mentira, lo que nos lleva, al mismo tiempo, al problema de la cabeza bien puesta" (Morin, 2001, 65).

En otras palabras, desde el espacio de la complejidad, hay que reconstruir una nueva gnoseología de lo fundamental en lo educativo, aupada en lo ético, lo estético y lo humanístico.

La tarea es recuperar la sensibilidad hacia lo humano, hacia el fratellos, hacia la formación de ciudadanos y a la utopía educativa de re-creación de sujetos políticos contemporáneos que privilegien el sujeto relacional y la alteralidad como una alternativa ${ }^{3}$.

3 A propósito, en el VII Encuentro del Foro Iberoamericano
En términos del español Roger Ciurana, hay una globalización que ilumina a pocos y deja a muchos a la sombra, abriendo brechas de marginación, llevando cada vez más a la degradación de la calidad de vida, de las solidaridades y al debilitamiento del sentido de la responsabilidad hacia los otros ${ }^{4}$, al anonimato del sujeto, debido a los avances tele-informáticos y tecnológicos que reducen las relaciones interpersonales al medio. Incluso, esta globalización ya no funciona, puesto que pregona un libre mercado que no es libre y, dado que es una globalización, bajo la dirección de un capitalismo especulativo financiero a la americana, no ha logrado materializar sus promesas de oportunidad y bienestar para todos. Las voces de miles de desilusionados han empezado a evidenciar que la globalización no ha aportado al mundo ninguno de los beneficios prometidos, sino el enriquecimiento de los más ricos o, mejor, de unos pocos. Además de Stiglitz, con sus textos El malestar de la globalización y La gran desilusión, a la lista de autores desilusionados con la globalización de mercado, se le han unido miles de voces, entre ellos la de gobernantes de varias naciones que han visto cómo sus esperanzas de un mundo mejor se desvanecen en el vacío ante un capitalismo especulativo financiero y una globalización de mercado excluyente.

Por ello, la educación está llamada a romper con la cultura enciclopédica, atomizada, tradicional, descontextualizada y fragmentada, para pensarse como una condición humana, capaz de enfrentar una racionalidad que ha reducido al sujeto a ser un instrumento para unos propósitos lucrativos, enajenantes y excluyentes, y a la humanidad y su necesaria humanización, a simples conceptos o ideales.

sobre Estrategias de Comunicación -FISEC-, celebrado en la ciudad de Cartagena de Indias, Colombia, los días 16 y 17 de septiembre de 2009, el tema central fue " $e l$ cambio en el sujeto: del actor racional al hombre relacional". En dicho encuentro, el filósofo Edgar Morín participó como conferenciante central. Se sugiere visitar la página de FISEC, www.fisec-estrategias.com.

4 Para ampliar estos conceptos, se sugiere ir al texto de Edgar Morin "Política de civilización". 
En tal sentido, la educación debe ser un sistema abierto y complejo que le dé un nuevo sentido y significado a lo que se enseña y al cómo se enseña, generando una organización y articulación del conocimiento contextualizado a las realidades complejas, a tener la capacidad de pensar lo complejo planetario, a profundizar en el abordaje de la realidad de una manera interdisciplinaria que realmente permita alcanzar el desarrollo humano en su multidimensionalidad.

A partir de estas nuevas significaciones, estaremos haciendo posible una educación que, unida a una política de civilización, "lleve a la toma de conciencia de la condición común a todos los humanos y de la diversidad de los individuos, de los pueblos, de las culturas, sobre nuestro arraigamiento como ciudadanos de la tierra" (Morin, 1999).

Según el premio Nobel de Economía en 1998. Amartya Sen, en su disertación sobre capital humano y capacidad humana, los beneficios de la educación son mayores para incrementar la capacidad humana, lo que hace que la persona sea más eficiente en la producción de bienes y, por supuesto, redunde en un mejoramiento del capital humano.

No obstante, lo más importante para Sen es que "si una persona se beneficia de la educación por la posibilidad de leer, argumentar, comunicar, elegir con mayor información, ser tenida en cuenta más seriamente por otros y así sucesivamente. (...) no es absurdo esperar que también pueda dirigir mejor su propia vida y tener más libertad para hacerlo. Ambas perspectivas ponen a la humanidad en el centro de la atención" (Sen, 1998, 70).

Desde esta perspectiva, el grupo de investigación Corpus de la Facultad de Comunicación de la Universidad d Medellín se propuso analizar el impacto de los egresados del programa de Comunicación y Relaciones Corporativas en las pequeñas y medianas empresas -pymes-, ubicadas en el municipio de Medellín (Colombia), en el período comprendido entre el 20022006, con el fin de establecer la incidencia del comunicador corporativo como sujeto político en estas entidades fundamentales para el desarrollo social.

La investigación pretendió dar respuesta a los interrogantes de pares académicos que, en distintos escenarios, han interrogado sobre el impacto social de los egresados de este programa de Comunicación que fue acreditado por tres años en febrero de 2006 y renovada su acreditación por seis (6) años en el 2009 por parte del Consejo Nacional de Acreditación y el Ministerio de Educación Nacional.

De igual forma, esta investigación representa una oportunidad para repensar no sólo la pro puesta curricular, sino el hacer y quehacer del comunicador y relacionista corporativo en entornos complejos y en el contexto de una utopía del desiderátum incumplido de la modernidad, es decir, de un mayor bienestar, progreso y desarrollo para todos en una sociedad democrática, desde la educación compleja.

\section{LA INVESTIGACIÓN EN CUESTIÓN}

El explorar, desde la fundamentación teórica que sustenta el programa de Comunicación y Relaciones Corporativas, los resultados del impacto generado por los egresados en las pequeñas y medianas empresas -pymes-, ubicadas en el municipio de Medellín, durante el período 2002-2006, resulta un imperativo, toda vez que este tipo de organizaciones se ha constituido en el principal motor de la economía a escala nacional y Medellín no es la excepción.

Se tomó como referente este período de tiempo, dado que en dicho cuatrienio no sólo se 
ha graduado un número importante de estudiantes del programa de Comunicación y Relaciones Corporativas, sino que también corresponde al período donde el Programa, la Facultad y la Universidad de Medellín realizaron cambios sustanciales al currículo y a una serie de acciones esenciales para el proceso formativo de sus egresados, tales como el proceso de renovación curricular, la adopción del sistema de créditos, las prácticas empresariales, la movilidad estudiantil, la internacionalización, entre otras.

Además, y dado el auge de los programas de Comunicación Social-Periodismo y de programas de Comunicación, en general, las empresas pequeñas y medianas de Medellín -pymes- están demandando cada vez más egresados del Programa a fin de que este tipo de profesional intervenga, en forma directa, sus procesos empresariales, no sólo desde el ámbito de la información, sino desde los conocimientos y habilidades comunicativas como apoyo a la labor gerencial.

Establecer el impacto de un programa de comunicación en un medio económico, caracterizado por cambios permanentes del entorno, resulta una necesidad, máxime si se entiende que la razón de ser de un programa de pregrado, en el contexto de la globalización y el desarrollo de una región, se constituye en ofertar contenidos curriculares que resuelvan, en la realidad, problemas sociales concretos en los ámbitos locales, regionales y nacionales, y en contextos internacionales.

En tal sentido, el programa de Comunicación y Relaciones Corporativas, que nació en 1995 y que arriba en el 2009 a catorce años (14) de existencia en el medio académico y productivo de la ciudad de Medellín, requiere conocer, de primera fuente, es decir, de sus egresados, el impacto que ha generado en dicho medio y, específicamente, en el sector de las pequeñas y medianas empresas -pymes-, las cuales se han convertido en el motor económico de la ciudad en los últimos años.
Si bien el período analizado, 2002-2006 corresponde a una de las etapas de existencia del Programa, su importancia resulta relevante, toda vez que las cohortes que egresaron incursionaron en el ámbito local en pequeñas y medianas empresas. Incluso, algunos de ellos no sólo fueron contratados por este tipo de organizaciones, sino que establecieron sus propias empresas, cuya clasificación corresponde a la categoría objeto de estudio de la investigación.

La pregunta orientadora resultó, entonces, simple y contunde, no sin antes aclarar que fue necesario establecer una serie de preguntas complementarias que sirvieran de elementos de introductorios y de ubicación a los encuestados.

\section{La pregunta orientadora correspondió a}

¿Cuál ha sido el impacto en los procesos de comunicación y desarrollo de las pequeñas y medianas empresas del municipio de Medellín en el período 2002-2006, generado por el trabajo de los comunicadores egresados del Programa de Comu nicación y Relaciones Corporativas de la Facultad de Comunicación de la Universidad de Medellín?

Este interrogante inspiró el proceso investigativo, pese a que fue necesario establecer otras preguntas complementarias, así como una conjetura guía, que no sesgaron la investigación, puesto que se privilegió el análisis de los datos arrojados, a través de ochenta y dos (82) encuestas, realizadas a egresados y estudiantes de último semestre con experiencia laboral que trabajan, directa o indirectamente, en las organizaciones estudiadas.

Por ello, este tipo de investigación no es concluyente y aplicable a todas las organizaciones, sino a aquellas donde se encuentran vinculados, directa o indirectamente, los egresados y estudiantes encuestados del programa de Comunicación y Relaciones Corporativas en el período estudiado. 
En la actualidad, las empresas poseen más y mejores tecnologías para los procesos multidireccionales de información y comunicación y también distintos medios de comunicación e información corporativa que implican que éstos sean conducidos por profesionales de la comunicación. Esta tendencia ha generado que las pymes contraten egresados de programas de Comunicación, y que uno de ellos sea el que corresponde al perfil del programa de Comunicación y Relaciones Corporativas de la Facultad de Comunicación de la Universidad de Medellín, ubicada en Medellín, Colombia.

Además de la pregunta orientadora y de las complementarias, y dado que la metodología de la investigación es del tipo descriptivo-cualitativo y retoma los elementos de la población a investigar, en forma descriptiva, a fin de acometer una acción interpretativa sobre los datos arrojados, se precisó de una conjetura guía que sirviera para el análisis. En tal sentido, dicha conjetura guía correspondió al siguiente postulado:

El egresado del Programa de Comunicación y Relaciones Corporativas está impactando el sector de las pequeñas y medianas empresas -PYMES-, ubicadas en el municipio de Medellín, de manera positiva para su desarrollo comunicativo, pero dicho impacto debería ser mayor, dado el creciente auge de la comunicación en las organizaciones y, en particular, en las correspondientes al objeto de estudio de esta investigación.

\section{ANÁLISIS DE LOS RESULTADOS}

En este artículo pretendemos mostrar los resultados de la investigación desde lo cuantitiativo y cualitativo. En primer lugar, se observó que los egresados en relación con los estudiantes encuestados están vinculados, de forma mayoritaria, al trabajo de pymes. El porcentaje de egresados (55\%) que trabaja en este tipo de organizaciones productivas es representativo y evidencia la acep- tación y vinculación de este tipo de profesionales en las pymes, hecho que no sucede en el público de estudiantes de último nivel, situación que lleva a inducir que aún los practicantes y las prácticas de la Facultad de Comunicación y del programa de Comunicación y Relaciones Corporativas, en particular, no han logrado penetrar de forma directa a los encargados de los procesos de selección de prácticas en las empresas.

Sin embargo, el hecho de que el número de egresados vinculados a pymes sea superior al porcentaje de estudiantes de último nivel resulta significativo. Según testimonios de los encuestados, cada vez más, los diferentes profesionales encargados de los procesos en las pymes le están dando reconocimiento e importancia a la comunicación. Estos datos permiten corroborar que la conjetura guía no se falsea, dado que el egresado del Programa está impactando, de manera positiva, la comunicación en las pymes y que el auge de la comunicación, como soporte a la dirección estratégica, es evidente, gracias al quehacer de los egresados que laboran en este tipo de organizaciones empresariales.

De otro lado, y como parte del análisis, es necesrio indicar que el porcentaje de $92.3 \%$ de estudiantes no vinculados a pymes contra un $7.7 \%$ de vinculados corresponde a una realidad del mercado. Sin duda alguna, la Universidad, la Facultad de Comunicación y los directivos del Programa, así como la coordinación de prácticas de la Universidad, deben hacer esfuerzos superiores por tratar de conseguir un número mayor de contactos con directivos de pymes o bien concienciar a aquellos, para que abran prácticas en este campo del saber, dada su importancia estratégica para las organizaciones productivas y de servicios, independiente de su tamaño.

En cuanto a sectores de desempeño en las pymes, los egresados realizan su labor en sectores de alimentos, servicios y transporte, 
principalmente. Los sectores de medios de comunicación y docencia corresponden a aquellos que reciben la labor de los egresados, hecho que resulta significativo, dado que estos sectores no corresponden a un número importante y significativo respecto a este tipo de organizaciones productivas, según se desprende de la estadística de la Cámara de Comercio para Medellín y Antioquia sobre el número de pymes existentes en Medellín y su Valle de Aburrá.

Resulta significativo también en el análisis de las encuestas observar el bajo porcentaje de egresados que laboran en el sector de textiles, hecho que evidencia la crisis por la que atraviesa este sector en la economía antioqueña, otrora sector emblemático en este mercado. Entre tanto, los estudiantes de último nivel son acogidos de manera mayoritaria por el sector de alimentos y servicios.

De la gráfica correspondiente a sector de pymes en las cuales están vinculados los estudiantes, resulta también significativo que los sectores de transportes, medios de comunicación y textiles no registran ningún tipo de vinculación de estudiantes practicantes, situación que deberá ser intervenida por la Universidad, toda vez que allí se registra un número representativo de las pymes existentes en la ciudad de Medellín y su Valle de Aburrá, según la estadística a la cual nos refererimos anteriormente.

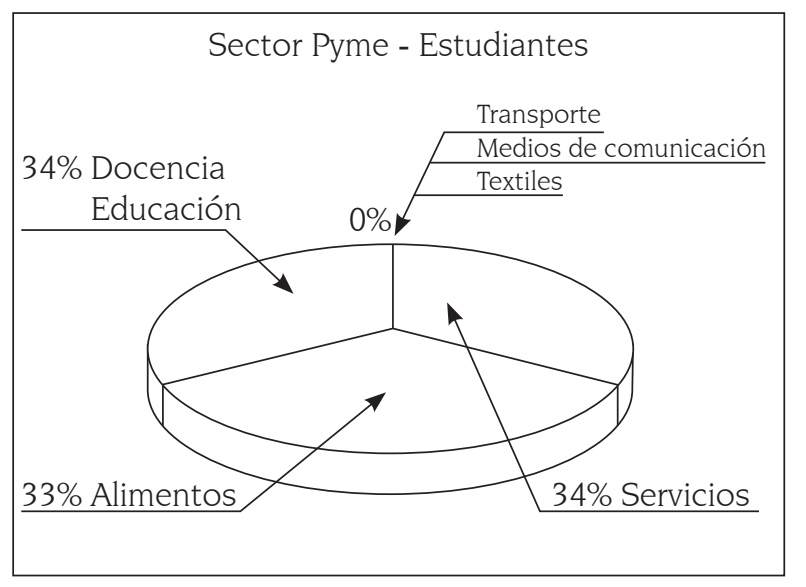

Entre tanto, la información relacionada con la vinculación de egresados al sector de las pymes se ve reflejada en el siguiente cuadro:

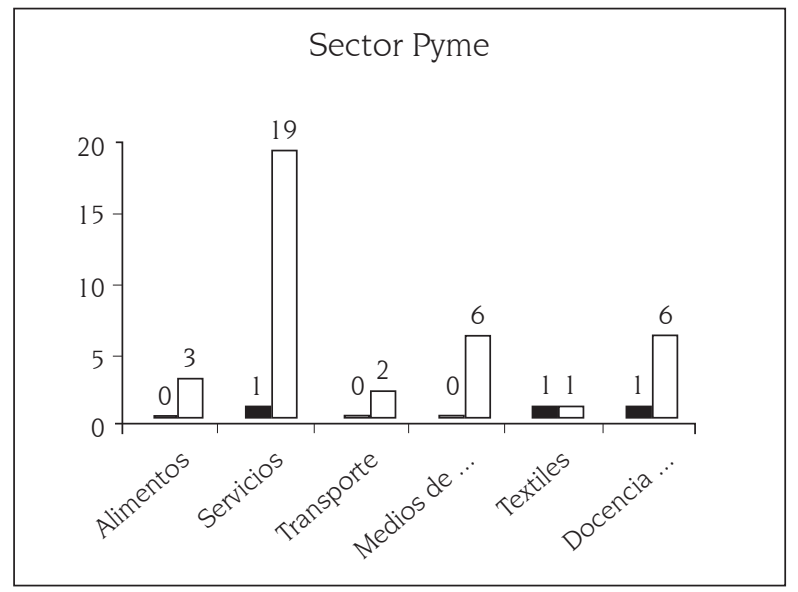

Del cuadro anterior, se infiere que la mayoría de las pymes que acogen la labor de egresados de comunicación corresponde a aquellas ubicadas en el sector servicios, situación que evidencia y corrobora lo que los economistas han llamado el paso de una economía precapitalista industrial en Medellín y su Valle de Aburrá a una economía terciarizada. Sin duda alguna, ya no es posible el calificativo de ciudad industrial de Colombia, pues dependemos cada vez más del sector servicios y de una economía basada más en la especulación financiera que en la producción y transformación de materias primas, características propias del capitalismo industrial que está representado, escasamente, por los tres países escandinavos (Suecia, Finlandia y Noruega), Japón, Corea del Sur y Alemania.

En el campo de las áreas profesionaleslaborales de desempeño, el correspondiente a la definición de públicos resulta ser el más representativo entre los encuestados, situación que se corresponde con el énfasis del programa de Comunicación y Relaciones Corporativas y su apuesta por formar un profesional dedicado a las relaciones con los públicos. De otro lado, y en el mismo nivel de importancia, los encuestados señalaron que la definición de perfiles, clientes, usuarios o 
públicos y diseños de estrategias para convocarlos; las estrategias de comunicación interpersonal y organizacional y la organización y logística de proyectos y certámenes organizacionales son las áreas en las cuales ellos se desempeñan en las pymes, respuestas que también se corresponden con el perfil de formación del Programa.

El cuadro siguiente corrobora no sólo la pregunta orientadora, es decir, un impacto real en las organizaciones estudiadas, sino también que permite actualizar la conjetura guía. De las respuestas, se infiere un impacto real y positivo en distintos ámbitos empresariales, así como un peso relativo de importancia del campo de la comunicación entre los diferentes actores que laboran en el interior de las pequeñas y mediadas empresas -pymes-, donde laboran los egresados y estudiantes de último nivel encuestados.

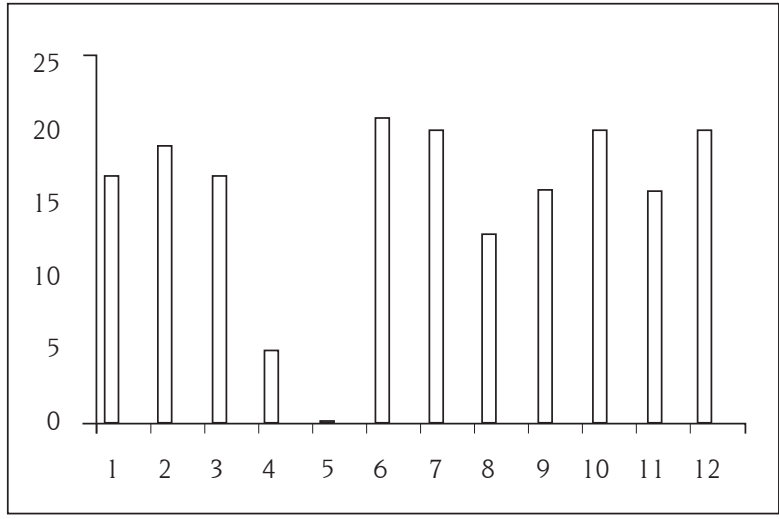

Columna 1: Realización de diagnósticos y pronósticos de comunicación interna y externa

Columna 2: Diseño de planes de comunicación

Columna 3: Formulación de procesos de comunicación

Columna 4: Evaluación sobre efectividad de procesos de comunicación

Columna 5: Auditoría de comunicaciones
Columna 6: Definición de públicos de un proyecto o producto

Columna 7: Definición de perfiles, clientes, usuarios o públicos y diseño de estrategias para convocarlos

Columna 8: Estrategias de medios de comunicación

Columna 9: Ventas

Columna 10: Estrategias de comunicación interpersonal y organizacional

Columna 11: Procesos de relaciones públicas

Columna 12: Organización y logística de proyectos y certámenes organizacionales

Del cuadro anterior, resulta interesante observar los bajos porcentajes obtenidos en las columnas 4 y 5, correspondientes a evaluación sobre la efectividad de procesos de comunicación y auditoría de comunicaciones, respectivamente. Esta situación refleja el poco interés por la evaluación y seguimiento por parte de los responsables de los procesos comunicacionales en las organizaciones estudiadas o bien de los directivos y el nivel de demandas que ellos exigen a los comunicadores contratados. Estas dos variables deben ser, sin duda alguna, más trabajadas en el campo de la formación de los comunicadores, máxime cuando en el mundo empresarial el tema de las auditorías y la evaluación cuentan con un alto nivel de aceptación y relevancia. De igual forma, se infiere que los temas relacionados con la evaluación y la auditoría en comunicación merecen mayor atención por parte de los empresarios de las pymes.

De manera puntual, y en las respuestas obtenidas, los encuestados señalaron, en el punto 
relacionado con "otros", aspectos o áreas, tales como: planes y asesorías en mercadeo, administración de recursos humanos, procesos de capacitación y alianzas. Sin embargo, y dado el nivel de dispersión, estas áreas profesionales y de desempeño laboral no registran cifras importantes en la gráfica de referencia.

En el análisis, resulta curioso que las columnas de las ventas y los procesos de relaciones públicas se encuentren en igual nivel de frecuencia en las respuestas de los encuestados. Decimos que curioso, dado que es de suponer que una de las mayores exigencias de los directivos de pymes correspondería, precisamente, al nivel de ventas y de relaciones con los públicos, clientes o usuarios que éstas deben manejar. Sin embargo, se infiere el auge y nivel de importancia que están alcanzando otras temáticas, distintas a las ventas, y más relacionadas con la comunicación en las organizaciones estudiadas o bien que los egresados y estudiantes de comunicación de último nivel han logrado diferenciar su quehacer específico entre los distintos profesionales que concurren en el interior de las organizaciones, objeto de estudio.

\section{PROMOCIÓN DE LA FORMACIÓN EMPRESARIAL}

Al indagar si la formación profesional recibida en el programa de Comunicación y Relaciones Corporativas y en la Facultad de Comunicación de la Universidad de Medellín estimula o promueve la formación empresarial, los encuestados tienen diferentes posturas. Un total de 19 egresados, por ejemplo, señalaron que Sí, mientras que un 23 que No. Entre tanto, veinte y cuatro (24) estudiantes, que aún están en su proceso formativo y que no tienen aún la categoría de egresados manifiestan que la formación recibida estimula y promueve la formación empresarial, mientras que 14 señalaron que No.

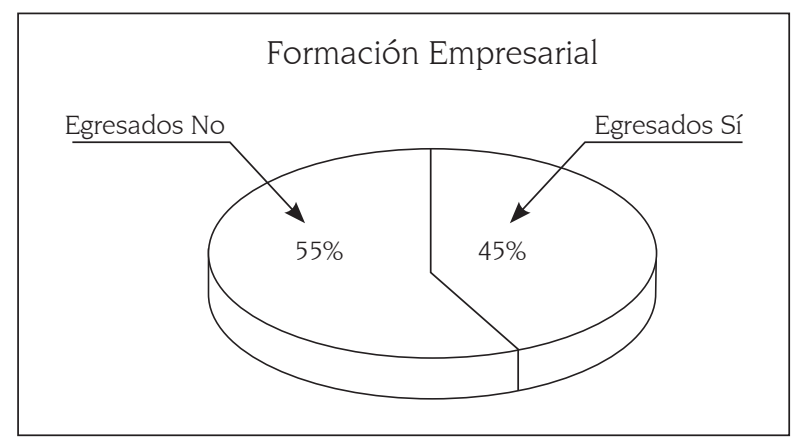

Al discriminar el número de egresados que respondieron Sí y los del No, la gráfica señala una diferencia de diez (10) puntos porcentuales, hecho que no representa una diferencia sustancial entre ambas respuestas. De esta situación particular se infiere que los egresados encuestados tienden a valorar menos su formación profesional en relación con el estímulo o promoción de formación empresarial. Cabe anotar que en el período estudiado, 2002-2006, el Programa contó con dos propuestas curriculares diferentes, hecho que justificaría el por qué de esta diferencia. Sin embargo, tanto el Programa, como la Facultad de Comunicación y la Universidad tratan de estimular dicha formación empresarial no sólo a través de la malla curricular, sino también con el ofrecimiento de diplomados en emprendimiento y la creación de un fondo de capital semilla, para el apalancamiento financiero a proyectos de empresa, generados por los estudiantes de últimos niveles y/o bien egresados de sus programas de pregrado.

Entre las justificaciones por el No, el común denominador de los egresados encuestados indican que, rara vez, los profesores proponen a los estudiantes trabajos académicos en los que se vean obligados a simular proyectos de emprendimiento. Por el contrario, dicen, que son constantes los ejemplos dados en clase del tipo "si su jefe le pide..." o "si el gerente de la empresa para la cual trabaja necesita...". De estas respuestas es pertinente sugerir que los directivos del Programa y de la Facultad de Comunicación y, de manera puntual, el programa de Egresados, adelanten acciones tendentes que los egresados tengan información 
de primer orden en relación con los diplomados de emprendimiento ofertados por la Universidad, a través del Centro de Intermediación Laboral para el egresado -CILAE-, adscrito a la Vicerrectoría de Extensión de la Universidad de Medellín.

Entre tanto, los estudiantes de último nivel, que poseen una mayor recordación de la propuesta curricular y de su nivel de formación, tienen una postura más favorable al indicar un 63\% de ellos que el Programa estímula la formación profesional, mientras que un $37 \%$ dice que No.

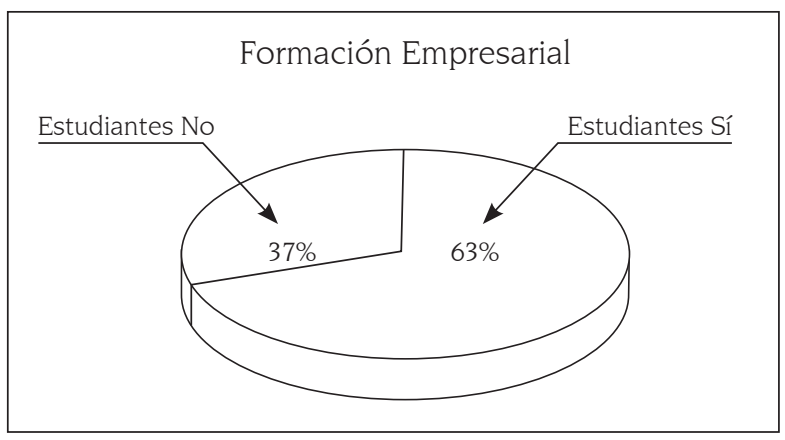

\section{ÁMBITO COMUNICACIONAL}

En los gráficos siguientes, los encuestados resaltan el hecho de que el ámbito comunicacional toma cada vez más importancia entre los directivos de las pequeñas y medianas empresas -pymes-. Tanto en la gráfica numérica como en las de porcentajes, los egresados y estudiantes de último nivel encuestados señalan la relevancia que toma la comunicación en las pymes. De las respuestas obtenidas de los escuestados que justifican el porqué, es pertinente señalar que la mayoría indica que en su trabajo han notado que uno de los elementos que menos importancia se le da en las pequeñas y medianas empresas es, precisamente, el de la comunicación corporativa. Esto se entiende porque al tener una infraestructura pequeña, se priorizan contrataciones de una manera funcional y pragmática por lo que resultan de mayor valor las labores que generan resultados inmediatos, tangibles y cuantitativos.
Sin embargo, los mismos encuestados dicen que entre los directivos de las Pymes ha cambiado, poco a poco, esta concepción y han empezado a entender la comunicación y, de manera puntual, la comunicación corporativa, como una forma de generar cierta coherencia entre lo que la empresa hace y lo que proyecta como una manera de apoyar, principalmente, la labor comercial.

De nuevo, estas últimas apreciaciones, detectadas y corroboradas en el trabajo de campo y en algunas de las justificaciones de los encuestados, sobre si en las pymes existe la posibilidad de desarrollo en el ámbito comunicacional, permiten validar la conjetura guía de la presente investigación.

En los gráficos siguientes, se evidencian unas diferencias mínimas en cuanto a la apreciación de los egresados y los estudiantes sobre la importancia dada por las pymes a los ámbitos propios de la comunicación y, en particular, por la comunicación corporativa.

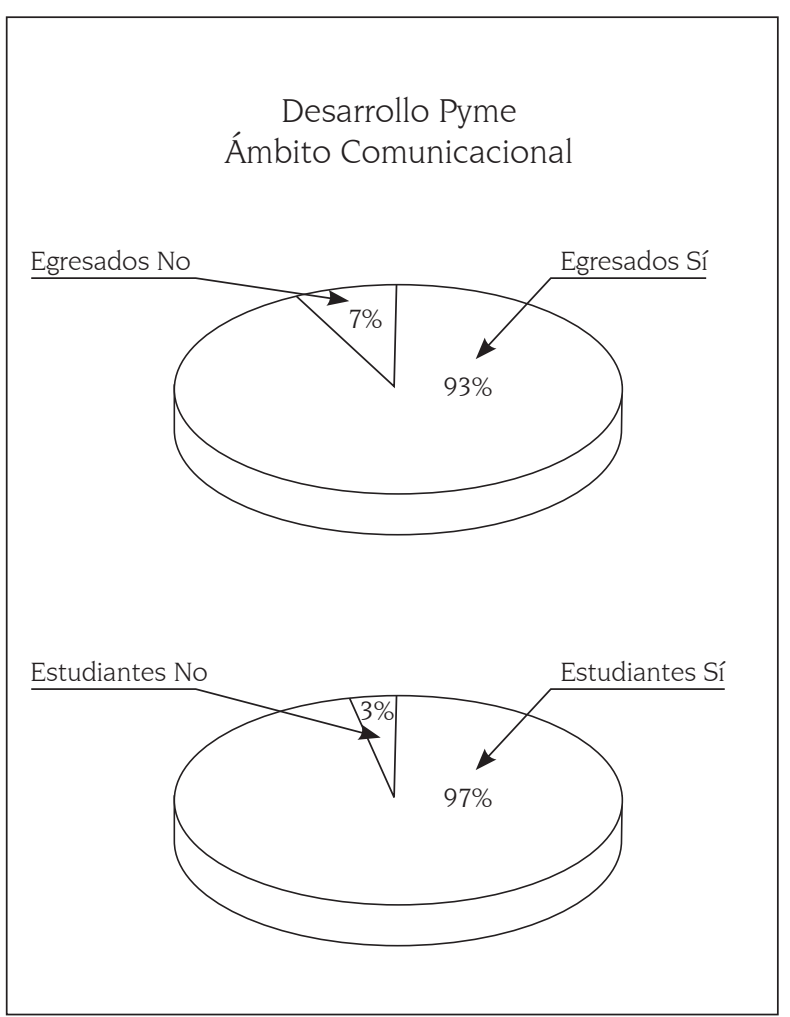




\section{DEMANDAS PARA IMPACTAR EL SECTOR DE LAS PYMES}

En las respuestas de los egresados y estudiantes de último nivel sobre cuáles cree que serían las demandas de un comunicador corporativo para impactar el sector de las pymes, las respuestas fueron disímiles, pero congruentes en cuanto a señalar temas atinentes al mercadeo, las nuevas tecnologías de la información y la comunicación, la creatividad, la innovación y el liderazgo.

En las respuestas se evidencia prelación por la formación comercial, con énfasis en el apoyo a ventas, dado que el interés de las pymes por estas materias es una prioridad. Otro aspecto relevante de las respuestas indica que la creatividad resulta fundamental para generar estrategias de comunicación innovadoras y ajustadas a los presupuestos que, en comparación con los de las grandes empresas, son muy reducidos.

Por las frecuencias de las respuestas, las siguientes son las temáticas que señalan los encuestados como prioritarias para su desempeño e impacto en las pymes donde trabajan:

- Dejar de lado la concepción de que sólo vale la pena vincularse a las grandes empresas

- Actualización permanente desde los perfiles profesiones

- Ser idóneos y estratégicos

- Logística

- Mercadeo y ventas

- Publicidad y diseño

- Manejo multimedial

- Incorporación de herramientas tecnológicas

- Creatividad y liderazgo

- Gestión- gerencia -manejo de personal - proveedores - nómina

- Relaciones públicas
- Transversalidad comunicacional

- Clima organizacional

- Disposición al cambio

- Imagen corporativa

- Posicionamiento

- Fidelización de públicos

- Habilidades comunicativas

- Salud ocupacional

- Normas de calidad

- Responsabilidad social

- Conocimiento en economía y finanzas

- Manejo de personal, proveedores y nómina

- Segundo idioma

- Asesoría y consultoría

- Implementación de modelos estratégicos que respondan a las PYMES

En atención a estas demandas, resulta pertinente señalar que se hace necesario ofertar diplomados, seminarios y/o cursos cortos que capaciten a los egresados y estudiantes en estas temáticas a fin de lograr un mejor y mayor desempeños de los comunicadores egresados del Programa en este importante sector de la economía.

\section{RECONOCIMIENTO LABORAL}

Al preguntarles a los encuestados sobre si en su experiencia dentro de las pymes fue reconocida su función como comunicador corporativo, un 75\% de éstos respondió que Sí, mientras que un 25\% dijo que No. Estas respuestas evidencian, sin duda alguna, el impacto que ha generado el Programa en las pymes y permite validar la conjetura guía.

La gráfica siguiente refleja tanto los porcentajes como el número de encuestados que respondieron de manera positiva a la pregunta: 
Reconocimiento laboral

Egresados No

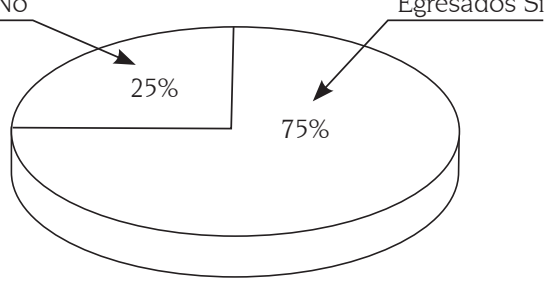

COMUNICACIÓN ESTRATÉGICA QUE AGREGA VALOR

\section{A LAS PYMES}

En relación con los aspectos de la comunicación estratégica que le agregan valor a las pymes para las que trabajan, los encuestados señalan varios, entre los cuales, y dado el número de frecuencia de las respuestas, los siguientes corresponden a los más destacados:

- Promoción de la pymes en los medios

- Publicity

- Canales de comunicación

- Manejo de público interno

- Clima organizacional

- Apoyo a recursos humanos

- Manejo de normas de calidad - Certificación

- Implementación de planes de comunicación (estrategias)

- Manejo de imagen corporativa

- Manejo de públicos y clientes

- Relaciones públicas

- Mercadeo

- Posicionamiento de marca

- Incorporación de herramientas tecnológicas

- Gestión de alianzas

De las respuestas puntuales, se rescatan varios testimonios, entre los cuales se destacan algunos que señalan que su aporte esencial en las pymes se corresponde con el desarrollo de una imagen constante con la identidad; en la definición de los mercados, según los productos y las diferentes estrategias para cautivarlos; en el sostenimiento de las relaciones con los clientes; en el estudio de las necesidades de las pymes en materia de comunicación y en la definición estratégica de los procesos de comunicación.

Al preguntarles por el hecho de si considera que los comunicadores corporativos tienen un significativo nivel de decisión dentro de las Pymes, un total de veintitrés egresados (23) y dos (2) estudiantes señalaron que Sí, mientras que nueve (9) egresados y un (1) estudiante dijeron que No.

En cuanto a porcentajes, tanto egresados como estudiantes son contudentes al afirmar que su trabajo es reconocido y que su participación en el nivel de decisión es relevante.

Las dos gráficas siguientes recogen, precisamente, los porcentajes correspondientes a las respuestas obtenidas:

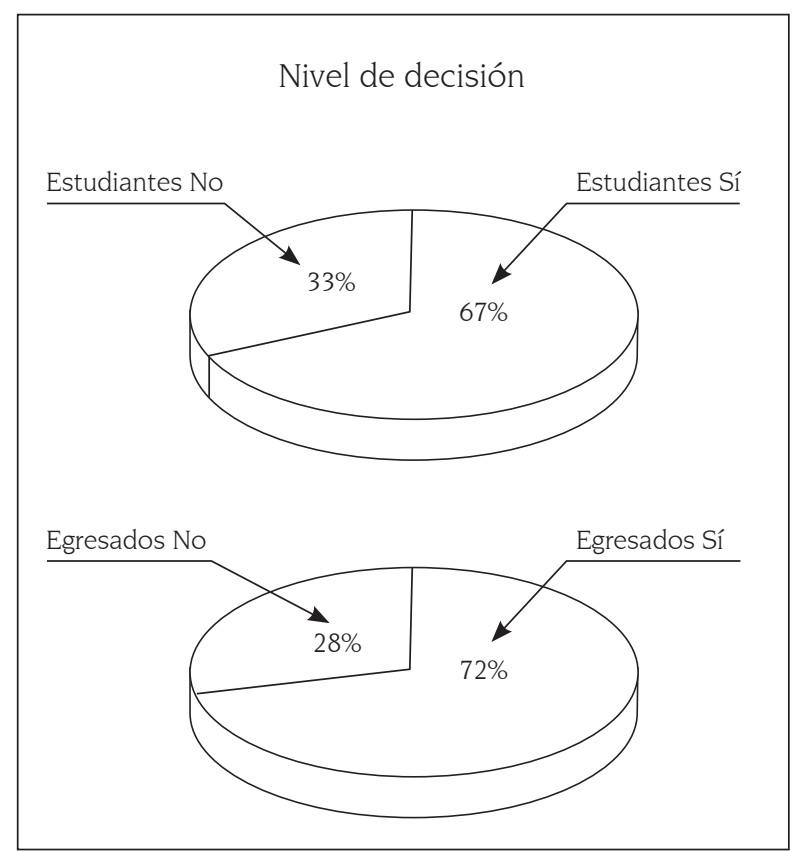


De los testimonios de los encuestados, recolectados en las libretas de apuntes del trabajo de campo, y por considerarlo relevante para la investigación, se destaca el siguiente:

Sí, porque normalmente si hay un comunicador vinculado a una Pyme, éste se encuentra en el nivel administrativo y si su trabajo es valorado, su opinión termina por volverse fundamental, que que cada estrategia que pretenda desarrollar la organización requiere ser comunicada y esto debe hacerse con sumo cuidado, para que no se pierda la armonía con los demás mensajes" (testimonio de uno de los encuestados).

El anterior testimonio resulta significativo, toda vez que la participación de los comunicadores en los niveles de decisión evidencia el alto grado de importancia que se le da al profesional de la comunicación en las pymes y al auge que toma la comunicación como un eje integrador y transversal a las organizaciones, sean éstas públicas o privadas, grandes, mediadas o pequeñas. El comunicador corporativo ha trascendido de su nivel instrumental a ser un eje político en la organización ${ }^{5}$.

\section{ESTRATEGIAS PARA EL CLIMA ORGANIZACIONAL EN LAS PYMES}

En cuanto a qué estrategias propondría para generar una cultura de la comunicación y las relaciones corporativas, que impacten el sector de las pequeñas y medianas empresas -pymes-, los encuestados señalaron varias, entre las cuales se destacan las siguientes:

- Manejar indicadores

5 Para una mayor ilustración sobre este tema recomendamos leer el texto "Construcción de identidad yfunción política en el discurso del director de comunicaciones", de Gladys Lucía Acosta y Jorge IgnacioSánchez. Sello Editorial Universidad de Medellín. ISBN 958-33-6675-7. Año 2004.
- Evidenciar resultados

- Utilizar las relaciones públicas

- Manejo de y con los medios

- Mercadeo y capacitación

- Organización de eventos

- Informar y comunicar procesos o proyectos

- Creación de clusters

- Realizar alianzas estratégicas

- Campañas de comunicación organizacional

- Seminarios y cursos para construir planes de comunicación para pymes

- Crear una agremiación para pymes

- Consultorías

- Implementación de los sistemas de calidad (regulación normativa)

- Responsabilidad social- Normativa

- Ética

- Liderazgo

- Pertinencia de formación en perfiles

- Idoneidad

- Conocimiento de la cultura y clima organizacional

- Posicionamiento de marca

- Imagen corporativa

- Implementación tecnológica

- Creación de departamentos de comunicación

Además de estas estrategias, los encuestadose señalan que es importante que las demás profesiones y profesionales que concurren en las pymes tengan un conocimiento estratégico de la comunicación, dado que el comunicador no puede lograr niveles adecuados de relación e interacción con administradores, economistas, abogados e ingenieros, entre otros, y que son interlocutores inmediatos en su labor en las pymes. 


\section{RETOS Y PROBLEMAS EN LAS PYMES}

Al indagar por el tipo de problemas a los que se ve enfrentado un comunicador y relacionista corporativo en las pymes, los encuestados señalan varios, entre los cuales se destacan los siguientes:

- Falta de credibilidad por parte de directivos empresariales

- Corto presupuesto para la consecución de recursos

- Desviación de funciones

- Desconocimiento de algunas pymes de la labor del comunicador y relacionista corporativo (posicionamiento profesional)

- Incertidumbre en el mercado

- $\quad$ Falta de trabajo en equipo

- Competitividad e idoneidad profesional

- Poca comprensión del ámbito comunicacional

- Rigidez organizacional

- Baja remuneración

Entre las respuestas obtenidas, el común denominador de los problemas corresponde a asuntos propios del desconocimiento por parte de los directivos de las pymes del papel y labor del comunicador en el apoyo a la gestión. La falta de cultura y conocimiento acerca de la comunicación y de las relaciones corporativas por parte de los empresarios es uno de los mayores problemas relacionados por los encuestados. Sin embargo, el tema de la comunicación está adquiriendo nivel de importancia tal como lo corroboran los propios encuestados en sus testimonios: "Se ha logrado mucho, pero todavía faltan muchas otras cosas por lograr".

Asimismo, la falta de presupuesto para el desarrollo de las propuestas comunicacionales sugeridas es uno de los problemas a superar por parte de los comunicadores de las pymes y, final- mente, un aspecto común en las respuestas señala que dado que la comunicación se constituye en un intangible y sus resultados no se pueden medir de manera inmediata o bien no se corresponden con la necesidad de medición cuantitativa que se privilegia en los ámbitos empresariales, resulta un asunto problémico superar este nivel de dificultad para el desarrollo y aplicación de estrategias de comunicación en las Pymes.

\section{NUEVAS TECNOLOGIAS INFORMÁTICAS}

Uno de los aspectos que quizá tenga mayor relevancia para el sector empresarial en el ámbito mundial tiene que ver con al manejo de la información, tanto en la parte documental como en la relación productiva.

La pequeñas y medianas empresas pymes no escapan de la necesidad de garantizar mayor eficiencia en los ámbitos competitivos. Se insiste en general que las necesidades en gestión documental son crecientes, y en el mercado colombiano, las disposiciones gubernamentales hacen que la digitalización y puesta en línea de documentos públicos y la información estatal creen oportunidades de crecimiento y expansión del negocio. Por ello, es indispensable que en los planes estratégicos de la pymes, se incorpore progresiva y visiblemente el valor de las nuevas tecnologías de la información y la comunicación.

A tenor de las alusiones de egresados y estudiantes, se infiere que es necesario que la Universidad, y en especial el programa de Comunicación y Relaciones Corporativas, respalde sus procesos formativos y profesionales con la adecuación e implementación de las tecnologías informáticas al servicio de las empresas, pues ven con preocupación que los servicios de gestión documental, apoyados en las nuevas tecnologías de la información y la comunicación, se constituyen en asunto prioritario para soportar las estrategias 
administrativas y comunicacionales de las empresas del sector.

Los comunicadores egresados y estudiantes replican que en Colombia y, en el mundo entero, las empresas crecen a un ritmo fuerte, y es preciso asumir sus ritmos para determinar las ventajas de la gestión documental, comunicativa e informacional que redunde en beneficio de procesos humanos y productivos de los distintos segmentos de las pymes. Las herramientas tecnológicas, al servicio de los comunicadores y de este tipo de organizaciones, exigen cada vez mayor profesionalismo y mayor productividad, porque ello les insta a implementar soluciones cada vez más creativas y eficientes, para los ritmos de competitividad que exigen estas organizaciones y sus públicos.

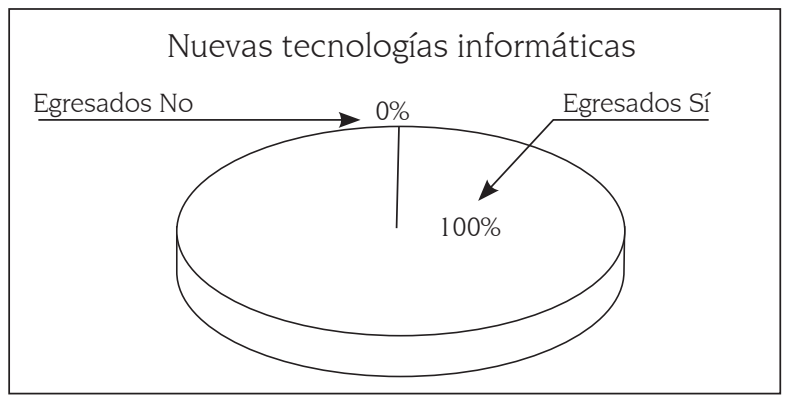

Precisamente, al preguntarles a los egresados por la contribución de los medios y las nuevas tecnologías informáticas a la gestión de impacto dentro de las pymes en las que laboran, éstos señalan un $100 \%$ el nivel de contribución, hecho que implica una necesaria consideración del programa de Comunicación y Relaciones Corporativas por dar respuesta a este tipo de demandas en la malla curricular.

Igual situación ocurre con los estudiantes de último nivel encuestados, quienes no dudan en establecer un porcentaje similar en su respuesta. Este hecho se correlaciona con la realidad actual de su proceso formativo, toda vez que hoy los estudiantes están más cometidos que antes a las nuevas tecnologías de la información y la comunicación, mejor conocidas como TIC.

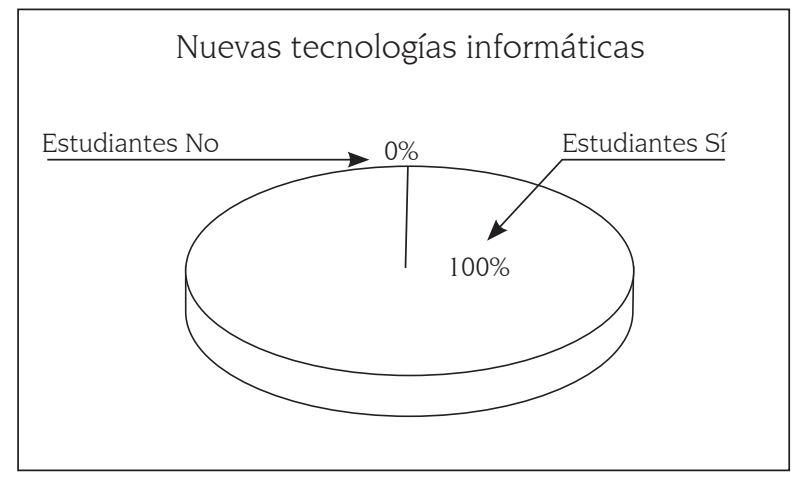

\section{CONCLUSIONES FINALES}

Si bien la investigación no corresponde a una de tipo concluyente y cuyos datos sean aplicables a todas las organizaciones, salvo a las seleccionadas, sus conclusiones pueden servir de referente, dado no sólo por el tipo de organizaciones seleccionadas (pymes), sino también por el número de egresados y la muestra seleccionada para la investigación.

De los datos descriptivos y cuantitativos, así como de la observación directa realizada a través del trabajo de campo en diferentes escenarios, entre ellos las reuniones mensuales convocadas por la coordinación del programa de egresados "Re-egresando a la U" de la Facultad de Comunicación, se concluye lo siguiente:

\section{Conclusión 1}

El programa de Comunicación y Relaciones Corporativas Sí está impactando de manera positiva a las pequeñas y medianas empresas de Medellín, en el período 2002-2006.

\section{Conclusión 2}

Este impacto se ve reflejado en el aporte que los comunicadores y relacionistas corporativas le vienen dando a la comunicación en las organizaciones estudiadas y, en particular, en el desarrollo de la imagen corporativa, la definición de mercados, el establecimiento de diferentes estrategias para mejorar las relaciones con los públicos, en el 
sostenimiento de las relaciones con los clientes y en el estudio de las necesidades de los mismos y en la definición estratégica de los procesos de comunicación.

\section{Conclusión 3}

El grado de impacto de la comunicación se corresponde con el nivel de importancia que el egresado y estudiante de último nivel le den a la comunicación y al trabajo en las pequeñas y medianas empresas. Dejar de lado la concepción de que sólo se puede vincular a la gran empresa es el primer paso para mejorar este impacto.

\section{Conclusión 4}

La creatividad y la innovación, ajustadas a los exiguos presupuestos de las pequeñas y medianas empresas - pymes-, resultan fundamentales, para que los egresados y estudiantes de último nivel logren impactar, de manera más contundente, a las organizaciones estudiadas.

\section{Conclusión 5}

La formación comercial, con énfasis en el mercadeo como apoyo a la función de ventas, resulta vital para la labor que adelanta el comunicador y relacionista corporativo en las pymes.

\section{Conclusión 6}

Para consolidar los procesos de formación y el nivel de correspondencia con la pertinencia en el ámbito empresarial, es necesario que el programa de Comunicación y Relaciones Corporativas oferte en su malla curricular asignaturas relacionadas con el campo de la administración (mercadeo, indicadores, presupuesto, liderazgo, trabajo en equipo, entre otros).

\section{Conclusión 7}

Para que el impacto de los egresados del programa de Comunicación y Relaciones Corporativas sea mayor, es necesario que se hagan esfuerzos mayores entre los directivos del Programa y de la Facultad, y la coordinación de prácticas de la
Universidad, a fin de concretar un número mayor de prácticas en las pequeñas y medianas empresas -pymes-, ubicadas en Medellín.

\section{Conclusión 8}

El impacto generado por los egresados y estudiantes de último nivel del programa de Comunicación y Relaciones Corporativas es evidente en el ámbito de la comunicación, pese al funcionalismo y pragmatismo que caracteriza a los directivos de las pequeñas y medianas empresas estudiadas.

\section{Conclusión 9}

La conjetura guía de esta investigación No se falsea. Por el contrario, se corrobora con las respuestas y los testimonios obtenidos por parte de los encuestados.

\section{Conclusión 10}

En la medida en que la comunicación logra penetrar distintos niveles jerárquicos en las organizaciones estudiadas, y en general, entre las profesiones diferentes a la comunicación, se logra un mayor impacto por parte de los egresados y estudiantes de último nivel que adelantan su labor comunicacional en las pymes.

\section{REFERENCIAS BIBLIOGRÁFICAS}

Abril, Gonzalo. (1997). Teoría general de la información. Madrid: Ediciones Cátedra.

Acosta, Gladys Lucía y Sánchez, Jorge Ignacio. (2004). Construcción de identidad y función política en el discurso del director de comunicaciones. Medellín: Sello Editorial Universidad de Medellín.

Aktouf, Omar. (2001). La administración: entre tradición y renovación. Cali: Universidad del Valle.

Allaire y Firsirotu. (1988). La naturaleza contractual de la planificación estratégica. Gestión. Vol. 13. № 2.

Allaire y Firsirotu. (1984). La stratégie en deux temps, trois mouvements. Gestión. Vol. 9, N² 2, abril. 
Andrews, K. y Christensen, W.G. (1965). Business policy, tests y cases. Illinois: Homewood.

Ansoff, H.I. (1971). Stratégie et développement de la entreprise. París: Editions Hommes et Tecniques.

Ansoff, H.I. (1976). From Strategic Planning to Strategic Management. Londrés, John Wiley \& Sons.

Bortolia, Annie. Comunicación y organización. (1992). Barcelona: Paidós.

Botero Montoya, Luis Horacio. (2007). Teoría de públicos. Lo público y lo privado en la perspectiva de la comunicación. Medellín: Sello Editorial Universidad de Medellín.

Botero Montoya, Luis Horacio y Galvis Ortiz, Carlos Alberto. (2009). Comunicación pública. Repensar la comunicación para la democracia. Medellín: Sello Editorial de la Universidad de Medellín.

Cámara de Comercio de Medellín para Antioquia. (2001). Antioquia avanza hacia nuevos esquemas de desarrollo empresarial. Documento. Mayo.

Cámara de Comercio de Medellín. (1999). Desarrollo económico local y regional en la era de la información. El caso de Medellín y el Área Metropolitana. Documento.

Chandler, A. (1972). Stratégie et structure. París: Editions de Organisation.

Corripio, Fernando. (1973). Diccionario etimológico general de la lengua castellana. Madrid: Tecnos.

Costa, Joan. (1995). Comunicación corporativa y revolución de los servicios. Madrid: Ediciones Ciencias Sociales.

. (1999). La comunicación en acción. Barcelona: Paidós.

(1998). La esquemática. Visualizar la información. Barcelona: Ediciones Paidós.

(2001). Imagen corporativa en el siglo XXI. Buenos Aires: La Crujía.

(2003). La imagen pública: una ingeniería social. Asociación Iberoamericana de Comunicación Estratégica -AICE-. Colección Hermes No 1.

y Moles, Abraham. Imagen didáctica. (1991). Barcelona: Enciclopedia del Diseño. Ediciones CEAC.
Danhke, G. L. (1998). Investigación y comunicación. Citado por Hernández Sampier, Roberto; Fernández Collado, Carlos y Baptista Lucio, Pilar. Metodología de la Investigación. México: McGraw Hill.

De Charles E. Osgood; Sebeok, Thomas. (1965). Psicolingüística: panorama de la investigación psicolingüística, 1954-1964. Barcelona: Editorial Planeta.

De Fleur, M.; Ball-Rokeach S.J.; (1993). Teorías de la comunicación de masas. Barcelona: Paidós.

Derrida, Jacques. (2002). La Universidad sin condición. Madrid: Editorial Trotta.

Drucker, Peter. (1958). The practice of Management. New York: Harper $\mathcal{E}$ Brothers.

Dufour, M. Síntesis. (1995). En Cuadernos de Administración $\mathrm{N}^{\circ} 20$ (mayo). Univalle.

Fernández E Dahnke. (1986). La comunicación humana: ciencia social. México: Editorial McGraw-Hill.

Fiske, John. (1984). Introducción al estudio de la Comunicación. Versión en español Bogotá: Editorial Norma.

Galeano Marín, Eumelia y Vélez, Olga Lucía. (2000). Investigación cualitativa. Estados del arte. Medellín: Digital Express.

Galindo C. Jesús, Lopera E. Egidio, Uribe de H. María Teresa y otros. 1993. Investigación cualitativa. Confrontación y perspectiva. Medellín: Universidad de Antioquia.

Garrido, Francisco. (2000). Comunicación estratégica. Barcelona: Gestión.

Imagen $E$ empresas. (1999). Santiago de Chile: Editorial Ril.

Comunicación Estratégica. (2000). En el Diario Financiero, Santiago de Chile, febrero.

. (2003). Comunicación, estrategia y empresa. Asociación Iberoamericana de Comunicación Estratégica-AICE-. Colección Hermes, № 2.

Glueck, W.F. (1976). Business policiy: strategy formation and management action. New Cork: Mcgraw-Hill.

Guber, Rosana. (2001). La etnografía: método, campo y reflexividad. Enciclopedia Latinoamericana de Sociocultura y comunicación. Bogotá: Editorial Norma. 
Guizar, M. Rafael. (1998). Desarrollo organizacional: principios y aplicaciones. México: McGraw Hill.

Hafsi, T. (1985). Du management au métamanagement: les subtilités du concept de stratégie. Gestión, vol 10, No 1, férvier.

Hallet, J. (1994). The New Role and Importance of Business Communication. EE. UU.: Editorial IABC, W..

Hernández Sampier, Roberto; Fernández Collado, Carlos y Baptista Lucio. (1998). Metodología de la Investigación. México: McGraw Hill.

Luhman, Niklas. (1987). Organización y decisión. Autopoiesis, acción y entendimiento comunicativo. Barcelona: Anthoropos.

Maletzke, Gerhard. (1976). Psicología de la comunicación. Quito: Editorial Época.

Mayo, Elton. (1977). Problemas sociales de una civilización industrial. Buenos Aires: Ediciones Nueva Visión.

Mcquail, Denis. (2000). Introducción a la teoría de la comunicación de masas. Barcelona: Paidós.

Mcquail, Denis. Windahl, Sven. (1984). Modelos para el estudio de la comunicación colectiva. Pamplona: Editorial Universidad de Navarra.

Mintzberg, H. (1979). "An Emerging strategy for direct research". Administrative Science Quarterly, vol 24. $N^{\circ} 4$, décembre.

Mintzberg, H. (1990). "La gestion est pas que une questions de chiffres". Le Press.

Morin, Edgar. (1992). El método, las ideas, cátedra. Documento. Madrid. pp. 132-154.

. (2001). La cabeza bien puesta. Repensar la reforma, reformar el pensamiento. Buenos Aires: Editorial Nueva Visión.

. Teoría y método. (1984). En Ciencia con consciencia. Barcelona: Anthropos.

Múnera Uribe, Pablo A. y Sánchez Zuluaga, Uriel H. 2004. Comunicación empresarial: una mirada corporativa. Medellín: Colección Hermes de Comunicación estratégica. AICE.

Newman, W.H. y Logan, J.P. (1965). Business policies and central management. Cincinnati: South-Weterns Pub.Co.
Neuman, J. Von y Morgenstern. (1974). Theory of gamnes and economic behavior. Princeton University Press.

Neumann, Elisabeth. (1995). La espiral del silencio. Opinión pública: nuestra piel social. Barcelona:

Pérez, Rafael. (2006). Estrategias de comunicación. Ciudad: Editorial Arial.

Pérez Suárez, Juan Manuel. (1997). Diccionario de Comunicaciones. Medellín: Universidad de Medellín.

Pizzolante N., Ítalo. (2001). El poder la comunicación estratégica. Caracas: Cograf Comunicaciones.

Porter, M. E. (1987). "The State of Strategic Thinking". The Economist. Vol 303. No 7499, 23 mai.

Restrepo J., Mariluz y Rubio Angulo, Jaime. (1994). Intervención en la organización. 2a ed. Bogotá: Significantes de Papel Ediciones.

Rogers, Everett M.; Shoemaker, Floyd. F. (1971). La Comunicación de innovaciones: un enfoque transcultural. México: Editorial Herrera.

Sánchez Z., Uriel Hernando. (2006). Modelos y esquemas de comunicación: algunos acercamientos. $2^{\mathrm{a}}$ ed. Medellín: Sello Editorial Universidad de Medellín.

Sanz De La T., Luis. (1994). Integración de la identidad y la imagen de la empresa. Madrid: ESIC Editorial.

Schramm, Wilbur. (1996). La ciencia de la comunicación. México: Editorial Robles.

Serrano, Manuel Martín. (2004). La producción social de la comunicación. $3^{\mathrm{a}}$ ed. Ciudad: Alianza Editorial.

Shannon, Claude E; Warren, Weaver. (1949). Matematical Theory of Communication. USA: Illinois University.

Stiglitz, Joseph. (2003). Cuando el capitalismo pierde la cabeza. México: Fondo de Cultura Económica.

Suárez, Ana María, Galvis, Carlos y otros. (2008). Investigación en comunicación: vigencia y prospectiva. Medellín: Sello Editorial Universidad de Medellín.

Tejada, Luis. (1987). Gestión de la imagen corporativa. Bogotá: Editorial Norma.

Tompkins, Phillip. (1989). "Translating Organizacional Theory" en Handbook of Organizacional Communication. United States: Editorial Sage. 
Van Riel, Cees. (1997). Comunicación corporativa. Madrid: Prentice Hall.

Vásquez Rodríguez, Fernando. (2003). Más allá del ver está el mirar. En Rostros y máscaras de la comunicación. Colección Hermes de Comunicación Estratégica. Asociación Iberoamericana de comunicación estratégica. Medellín: AICE.

Vásquez Montalbán, Manuel. (2000). Historia y comunicación social. Barcelona: Mondadori.

Veron, Eliseo. (1995). Construir el acontecimiento. Barcelona: Gedisa.
Watzlawick, Paul; Veabin, Janet H y Jackson, Don D. (1967). Teoría de la comunicación humana: interacciones, patologías y paradojas. Buenos Aires: Editorial Tiempo Contemporáneo.

Weil, Pascale. (1990). La comunicación global. Barcelona: Editorial Paidós.

Wodak, Ruth y Meyer, Michael. (2001). Métodos de análisis crítico del discurso. Barcelona: Editorial Gedisa.

Wolf, Mauro. (1985). La investigación de la comunicación de masas: crítica y perspectivas. Barcelona: Paidós. 